\title{
KEYNOTE-010: flash of a supernova (immune-checkpoint inhibitors) in second-line non-small cell lung cancer
}

\section{Hidehito Horinouchi}

Department of Thoracic Oncology, National Cancer Center Hospital, Chuo-ku, Tokyo, Japan

Correspondence to: Hidehito Horinouchi, MD, PhD. Department of Thoracic Oncology, National Cancer Center Hospital, 5-1-1, Tsukiji, Chuo-ku, Tokyo 1040045, Japan. Email: hhorinou@ncc.go.jp.

Provenance: This is an invited Editorial commissioned by Section Editor Dr. Shangwen Dong (Thoracic Oncology, Journal of Thoracic Disease, Tianjin Medical University General Hospital, Tianjin, China).

Comment on: Herbst RS, Bass P, Kim DW, et al. Pembrolizumab versus docetaxel for previously treated, PD-L1-positive, advanced non-small-cell lung cancer (KEYNOTE-010): a randomised controlled trial. Lancet 2016;387:1540-50.

Submitted Sep 18, 2017. Accepted for publication Oct 16, 2017.

doi: $10.21037 /$ jtd.2017.10.135

View this article at: http://dx.doi.org/10.21037/jtd.2017.10.135

The KEYNOTE 021 trial was a randomized phase II/III trial comparing docetaxel and pembrolizumab as a secondline treatment for non-small cell lung cancer (NSCLC) in which 202 institutions from 24 countries participated (1). Eligible patients were as follows: progression after treatment with platinum combination therapy, EGFRTKI or AKL-TKI; an age of 18 years or older; an Eastern Cooperative Oncology Group Performance Status (ECOGPS) of $0 / 1$; and programmed death-ligand 1 (PD-L1) with a tumor proportion score (TPS) of $1 \%$ or more. Subjects were assigned to equally numbered treatment groups in an unblinded manner and received $2 \mathrm{mg} / \mathrm{kg}$ of pembrolizumab, $10 \mathrm{mg} / \mathrm{kg}$ of pembrolizumab, or $75 \mathrm{mg} / \mathrm{m}^{2}$ of docetaxel. Pembrolizumab was administered over 30 minutes every 3 weeks for as long as 24 months. The allocation was adjusted according to PS (0/1), region (East Asia/Non-East Asia), and PD-L1 TPS. The primary endpoint was overall survival (OS) for all eligible patients and for the subgroup with a PD-L1 TPS of $50 \%$ or more. The objective response rate (ORR) and treatment duration were set as secondary endpoints. Assuming a hazard ratio (HR) of 0.6, a total of 1,460 patients were required, taking the proportion of $\mathrm{PD}$ L1-positive patients into account. In the subgroup with a PD-L1 TPS of $50 \%$ or more, the assumed OS HR was 0.55 with an $81 \%$ power (one-sided $\alpha=0.00825$ ). In the entire population, the assumed OS HR was 0.70 with an $80 \%$ power (one-sided $\alpha=0.001$ ).

This important trial revealed three key points: the efficacy of pembrolizumab in comparison with docetaxel, the prevalence of PD-L1 expression in a huge NSCLC population, and the dose setting for pembrolizumab.

First of all, in terms of efficacy, the superiority of pembrolizumab over docetaxel in terms of the OS time was shown for both the PD-L $1 \geq 50 \%$ and PD-L $1 \geq 1 \%$ populations. In the $2-$ and $10-\mathrm{mg} / \mathrm{kg}$ subgroups, the HRs were $0.71(0.58-0.88, \mathrm{P}=0.0008)$ and $0.61(0.49-0.75$, $\mathrm{P}<0.0001)$, respectively. The median OS time was 10.4 months in the $2-\mathrm{mg} / \mathrm{kg}$ group, 12.7 months in the $10-\mathrm{mg} / \mathrm{kg}$ group, and 8.5 months in the docetaxel group. The ORRs were $18 \%, 18 \%$ and $9 \%$, respectively. Furthermore, in the group with a PD-L1 TPS of $50 \%$ or more, the OS HRs were 0.54 $(0.38-0.77, \mathrm{P}=0.0002)$ and $0.50(0.36-0.70, \mathrm{P}<0.0001)$ for the 2 - and $10-\mathrm{mg} / \mathrm{kg}$ subgroups, respectively. The median OS was 14.9 months in the $2-\mathrm{mg} / \mathrm{kg}$ group, 17.3 months in the $10-\mathrm{mg} / \mathrm{kg}$ group, and 8.25 months in the docetaxel group. The ORRs were $30 \%, 29 \%$ and $8 \%$, respectively.

To evaluate PD-L1 as a biomarker, clone $22 \mathrm{C} 3$ was used. Archived specimens were initially accepted; however, a new tissue sampled obtained during a biopsy performed immediately prior to trial registration became mandatory after the randomization of 456 patients. Also, after 441 patients had been randomized, the allocation was adjusted according to either a PD-L1 TPS of $1-49 \%$ or $50 \%$ or more. As a result, 2,699 patients were registered in this study, a PD-L1 expression analysis was performed for 2,222 patients, 1,475 (66\%) were positive for PD-L1 TPS with an 
Table 1 Efficacy results of major phase III trials (nivolumab, pembrolizumab, atezolizumab)

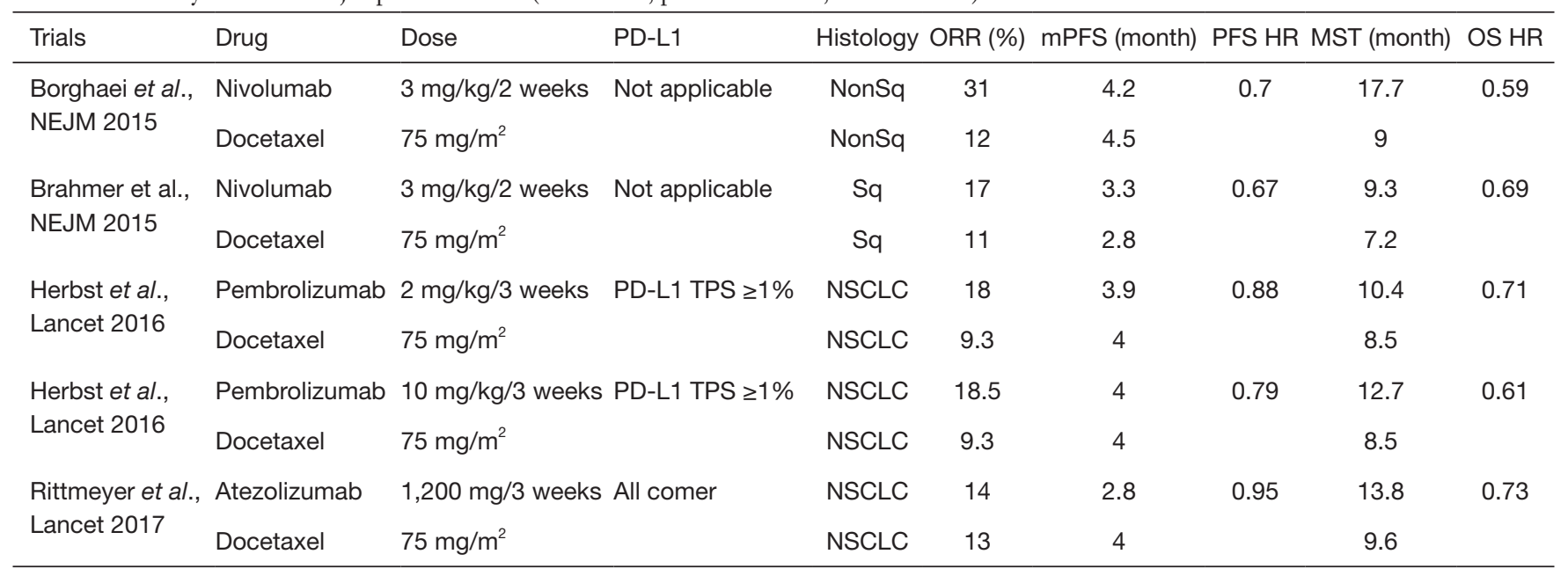

PD-L1, programmed death-ligand 1; TPS, tumor proportion score; ORR, object response rate; PFS, progression-free survival; mPFS, median progression-free survival; HR, hazard ratio; MST, median survival time; OS, overall survival; NSCLC, non-small cell lung cancer.

expression of $1 \%$ or more, and $633(28 \%)$ were positive for PD-L1 TPS with an expression of $50 \%$ or more. Finally, 1,034 people $(70 \%)$ were registered after selecting 1,475 patients with a TPS of $1 \%$ or more according to the study's eligibility criteria.

In this study, two levels ( 2 and $10 \mathrm{mg} / \mathrm{kg}$ ) of pembrolizumab were studied. This interesting dose setting (actually 5 times wider in range) was based on previously reported results for the PK/PD analysis of pembrolizumab. According to the previous study, the minimum dose for which the antitumor activity of pembrolizumab could be anticipated was $2 \mathrm{mg} / \mathrm{kg}$ (2). The KEYNOTE 010 trial was conducted to verify the clinical significance of these two dose settings. As a result, the response rates were 18\% vs. $18 \%$ in the population with a TPS of $1 \%$ or more and $30 \%$ vs. $29 \%$ in the population with a TPS of $50 \%$ or more. Furthermore, no clear survival difference was seen regardless of the TPS. Based on this result, $2 \mathrm{mg} / \mathrm{kg}$ was subsequently used as the standard dose.

The breakthrough of immune checkpoint inhibitors in NSCLC is remarkable. In both the CheckMate 017 and the CheckMate 057, nivolumab was superior to docetaxel regardless of the expression of PD-L1 (Table 1) $(3,4)$. In the OAK trial, atezolizumab surpassed docetaxel regardless of the expression of PD-L1 (5). Pembrolizumab was superior to docetaxel in the KEYNOTE 010. At the same time, the results of the REVEL trial, in which docetaxel + ramucirumab outperformed docetaxel monotherapy, have been reported (6). The 15-year era of using docetaxel as a second-line treatment for NSCLC has recently come to an end. Regarding the effectiveness of immune checkpoint inhibitors, the expression of PD-L1 is considered to be the most influential biomarker at present. The KEYNOTE 010 trial contained a patient population with a PD-L1 TPS of more than 1\%; however, the CheckMate 017, CheckMate 057 , and OAK trials included patients regardless of PDL1 expression. Among the PD-L1 antibody clones that have been adopted, $22 \mathrm{C} 3$ for pembrolizumab has been used as a companion diagnostic, and 28-8 for nivolumab and SP-142 for atezolizumab have been developed as complementary diagnostics. As a result, the superiority of immunity checkpoint inhibitors over docetaxel has been confirmed regardless of PD-L1 expression. In contrast, in the population with a PD-L1 TPS of $50 \%$ or more, pembrolizumab outperformed platinum combination therapy in the KEYNOTE 024 trial. Thus, researchers have been confused by a lack of consistency in terms of the value of PD-L1 expression as a biomarker for immunecheckpoint inhibitors.

Regarding safety, the CheckMate 017, CheckMate 057, KEYNOTE 010, and OAK trials have consistently shown a good trend for immune checkpoint inhibitors, compared with docetaxel (Table 2). The incidences of grade 3 and higher adverse events are reported to be $7-10 \%$ for nivolumab, $13-16 \%$ for pembrolizumab, and $15 \%$ for atezolizumab, making these agents safer than docetaxel. Regarding the difference in safety among immune checkpoint inhibitors, nivolumab tends to be 


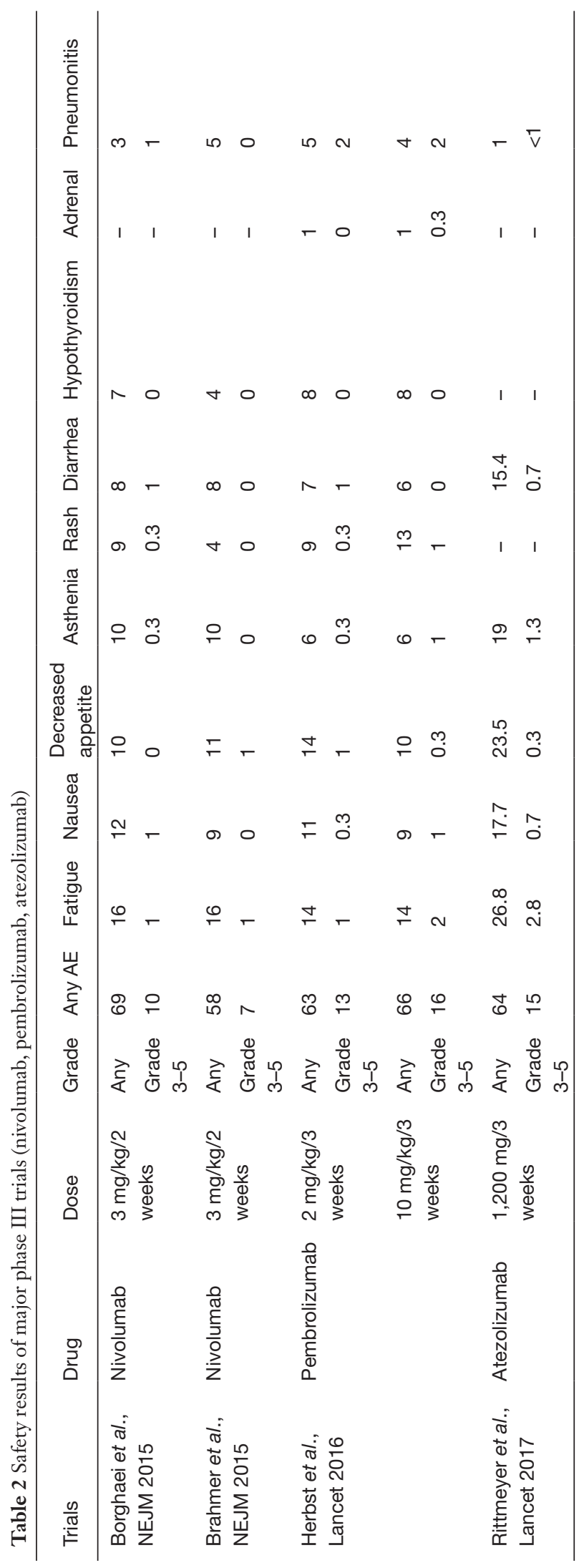

associated with a slightly lower frequency of grade 3 or higher adverse events. In addition, attention should be paid to the frequency of immune-related adverse events, such as thyroid dysfunction, adrenal insufficiency, and pneumonitis, which are characteristic of immune checkpoint inhibitors.

For the second-line treatment of NSCLC, several phase III trials, including the KEYNOTE 010 trial, have resulted in the triumph of immune checkpoint inhibitors over docetaxel. This is a remarkable achievement in medical history; however, as already mentioned, pembrolizumab has become the standard therapy for the primary treatment of patients with a PD-L1 TPS of $50 \%$ or more. Although the KEYNOTE 021 was a phase II trial, it revealed that pembrolizumab in combination with a platinum-doublet provided superior results than the platinum-doublet alone (7). As a result, the FDA approved pembrolizumab for the firstline treatment of patients with a PD-L1 TPS of $50 \%$ or greater and pembrolizumab plus platinum combination therapy in patients regardless of PD-L1 expression. The position of immune checkpoint inhibitors is quickly shifting from a second-line setting to a first-line treatment. The time to savor the brilliant achievements in the battlefield of second-line treatments may be unexpectedly short.

\section{Acknowledgements}

Research Grants: MSD, Merck serono, Chugai, Novartis, Astellas, Ono pharmaceutical.

\section{Footnote}

Conflicts of Interest: H Honoraria-Lilly, Kyowa-Kirin, Chugai, Ono pharmaceutical, Bristol-Myers Squibb, Novartis.

\section{References}

1. Herbst RS, Baas P, Kim DW, et al. Pembrolizumab versus docetaxel for previously treated, PD-L1-positive, advanced non-small-cell lung cancer (KEYNOTE-010): a randomised controlled trial. Lancet 2016;387:1540-50.

2. Lindauer A, Valiathan CR, Mehta K, et al. Translational Pharmacokinetic/Pharmacodynamic Modeling of Tumor Growth Inhibition Supports Dose-Range Selection of the Anti-PD-1 Antibody Pembrolizumab. CPT Pharmacometrics Syst Pharmacol 2017;6:11-20.

3. Borghaei H, Paz-Ares L, Horn L, et al. Nivolumab versus Docetaxel in Advanced Nonsquamous Non-Small-Cell 
Lung Cancer. N Engl J Med 2015;373:1627-39.

4. Brahmer J, Reckamp KL, Baas P, et al. Nivolumab versus Docetaxel in Advanced Squamous-Cell Non-Small-Cell Lung Cancer. N Engl J Med 2015;373:123-35.

5. Rittmeyer A, Barlesi F, Waterkamp D, et al. Atezolizumab versus docetaxel in patients with previously treated non-small-cell lung cancer (OAK): a phase 3, openlabel, multicentre randomised controlled trial. Lancet 2017;389:255-65

6. Garon EB, Ciuleanu TE, Arrieta O, et al. Ramucirumab

Cite this article as: Horinouchi H. KEYNOTE-010: flash of a supernova (immune-checkpoint inhibitors) in second-line nonsmall cell lung cancer. J Thorac Dis 2017;9(11):4187-4190. doi: 10.21037/jtd.2017.10.135 plus docetaxel versus placebo plus docetaxel for secondline treatment of stage IV non-small-cell lung cancer after disease progression on platinum-based therapy (REVEL): a multicentre, double-blind, randomised phase 3 trial. Lancet 2014;384:665-73.

7. Langer CJ, Gadgeel SM, Borghaei H, et al. Carboplatin and pemetrexed with or without pembrolizumab for advanced, non-squamous non-small-cell lung cancer: a randomised, phase 2 cohort of the open-label KEYNOTE-021 study. Lancet Oncol 2016;17:1497-508. 